\title{
Simulating the Majorana dynamics with ultracold atomic gases in a bilayer honeycomb lattice
}

\author{
Xin Shen, ${ }^{1,2}$ Dan-Wei Zhang, ${ }^{1}$ Hui Yan, ${ }^{1}$ Zhi Li,,${ }^{1, *}$ and Shi-Liang Zhu ${ }^{1,3}$ \\ ${ }^{1}$ Guangdong Provincial Key Laboratory of Quantum Engineering and Quantum Materials, School of Physics \\ and Telecommunication Engineering, South China Normal University, Guangzhou 510006, China \\ ${ }^{2}$ Shenzhen Institute for Quantum Science and Engineering, and Department of Physics, Southern University of Science and Technology, \\ Shenzhen 518055, China \\ ${ }^{3}$ National Laboratory of Solid State Microstructures and School of Physics, Nanjing University, Nanjing 210093, China
}

(Received 18 June 2019; published 10 January 2020)

\begin{abstract}
Cold atomic gases in a hexagonal optical lattice is a highly effective quantum simulator for exploring graphenelike physics. In this paper, we propose a feasible scheme to simulate and detect the Majorana dynamics with cold atoms in a bilayer hexagonal lattice. We explore the dynamics of a general Majorana particle, in which three key observables featuring the Majorana dynamics are studied, which include the pseudoenergy, orthogonality, and the fidelity. The results reveal that the fidelity is a good observable to distinguish Majorana from Dirac or Weyl dynamics. Through the decomposition of the Majorana equation, we address the Majorana Zitterbewegung, which is indeed the addition of two Dirac Zitterbewegung. Finally, we present a method to detect the Majorana dynamics with the quantum-state tomography implemented with quenches. Our scheme may provide an avenue for observing the phenomena described by the Majorana equation through bilayer graphene lattices.
\end{abstract}

DOI: 10.1103/PhysRevResearch.2.013037

\section{INTRODUCTION}

In 1937, Italian theoretical physicist Ettore Majorana noticed that the solution of the Dirac equation could be totally real on the condition that the Dirac gamma matrices are chosen to be pure imaginary. Then the particle described by the wave function serves meanwhile as the antiparticle of itself [1]. The equation is called the Majorana equation. Soon afterwards, researchers found that it does not necessarily need pure imaginary gamma matrices to establish the Majorana equation [2]. The more general Majorana equation (GME) describes a type of novel particles beyond the standard model. To directly investigate the dynamics of such Majorana particles is an impossible mission owing to the fact that the particle wave function and its charge conjugation are both found in the equation [3]. Researchers are driven to look for other ways to study the GME. In particular, experimental and theoretical efforts have been devoted to the quantum simulation of the GME, through which powerful and feasible schemes have been finally formed in trapped ion [4-6] and optical waveguide [7] systems.

On the other hand, as one of the promising quantum simulators [8-12], the cold atomic lattice systems have been well proven reliable on the simulation of relativistic quasiparticles. With several proposed lattice schemes, noteworthy progress

\footnotetext{
*lizhiphys@126.com

Published by the American Physical Society under the terms of the Creative Commons Attribution 4.0 International license. Further distribution of this work must maintain attribution to the author(s) and the published article's title, journal citation, and DOI.
}

has been made in simulating relativistic phenomena with mono- or multilayer honeycomblike sheets [13-17]. Klein paradox, Zitterbewegung, and relativistic Landau levels have been realized in tabletop ultracold atomic experiments one by one [18-26]. Furthermore, the recent finding of merged Dirac points [27,28], Weyl semimetal [29-36], one-dimensional (1D) and two-dimensional (2D) spin-orbit couplings [37-40] have given a great push for the further understanding of relativistic effects and relevant phenomena in condensed-matter and high-energy physics [15]. The artificial gauge fields for neutral atoms also constitute an effective platform [11,4145] for simulation of the relativistic quasiparticle and related topological states [8-12]. The well-known Harper-Hofstadter model [46-48], Haldane model [49,50], and other related models being realized one by another, and some topological features such as chiral edge states [51,52], the Berry phase, and Chern numbers $[41,53]$ have been observed in cold atomic systems.

So far, much attention has been paid to the study of Dirac/Weyl physics in the above graphenelike systems. Nevertheless, only a few papers have discussed the Majorana physics [4-7]. This paper is devoted to simulating the dynamics of a general Majorana particle (GMP) in the cold-atom system. Three crucial observables in differentiating Majorana from Dirac/Weyl physics, i.e., pseudoenergy, orthogonality, and fidelity, will be investigated. Theoretically, the GMP can be decomposed into two Majorana fermions with opposite masses. We propose a feasible scheme to realize the Majorana equations with an $A A$-stacked bilayer of hexagonal lattice. The proposed lattice preserving inversion symmetry generates a doubly degenerate energy spectrum while the Dirac dispersion at the Brillouin corner consists of opposite mass terms. The final GMP state can be constructed via tunable interlayer 
coupling so that the measurement is based on conventional quantum-state tomography in a single layer. In the cold-atom system, the final state tomography can be experimentally implemented via the quench dynamics, which was developed for the measurement of the Chern number [54,55].

The paper is organized as follows. In Sec. II, we present the model for simulating the GME. In Sec. III, we investigate the observables in Majorana dynamics with atomic wave packets. In Sec. IV, we propose an experimental procedure and measurement schemes for simulating Majorana dynamics in the tabletop atomic lattice system. The paper is concluded in Sec. V.

\section{BILAYER HEXAGONAL OPTICAL LATTICE}

The GME in general suffers a nonunitary evolution due to the existence of an unphysical complex conjugate operator. However, in two spatial dimensions one can circumvent this by decomposing the GMP into two Majorana ferimions, which enables the experimental investigation of the GME $[4,7]$. To be explicit, we start from the $(2+1)$-dimensional two-component GME which has the form

$$
i \partial_{t} \Psi=\left(p_{x} \sigma_{x}+p_{y} \sigma_{y}\right) \Psi-i M \sigma_{y} \Psi^{*},
$$

where $\sigma_{x, y}$ are the usual Pauli matrices and the superscript* denotes the complex conjugate. By decomposing $\Psi=\left(\psi_{1}+\right.$ $\left.i \psi_{2}\right) / \sqrt{2}$ and assuming the Majorana fermion condition $\phi_{\mu}=$ $-i \sigma_{z} \sigma_{y} \phi_{\mu}^{*}$, one obtains

$$
i \partial_{t}\left(\begin{array}{l}
\psi_{1} \\
\psi_{2}
\end{array}\right)=\left[\begin{array}{cc}
h_{1}(\mathbf{p}) & 0 \\
0 & h_{2}(\mathbf{p})
\end{array}\right]\left(\begin{array}{l}
\psi_{1} \\
\psi_{2}
\end{array}\right),
$$

where $h_{1,2}(\mathbf{p})=\left(p_{x} \sigma_{x}+p_{y} \sigma_{y}\right) \pm M \sigma_{z}$ describe the corresponding two Majorana fermions. Through this procedure, one obtains a Hermitian Hamiltonian consisting of two decoupled Dirac equations with opposite masses. Hence the simulation of the GME can be mapped as the simulation of the two Dirac equations, which covers the rest of the paper. In the rest of this section, we shall first present an optical lattice scheme for the realization of these two Dirac Hamiltonians.

Inspired by the experimental achievements in simulating Dirac quasiparticles [13,27], here we propose an $A A$-stacked bilayer optical lattice with each layer formed by three retroreflected laser beams of wavelength $\lambda$. The lattice potential is given by [27]

$$
\begin{aligned}
V_{\mu}(x, y)= & -V_{\bar{X}} \cos ^{2}\left(k_{L} x+\theta / 2\right)-V_{X} \cos ^{2}\left(k_{L} x\right) \\
& -V_{Y} \cos ^{2}\left(k_{L} y\right) \\
& -2 \alpha \sqrt{V_{X} V_{Y}} \cos \left(k_{L} x\right) \cos \left(k_{L} y\right) \cos \left(\varphi_{\mu}\right)
\end{aligned}
$$

where the subscript $\mu=1,2$ denotes the first and second layers. $\alpha$ is the visibility of the interference pattern and $k_{L}=$ $2 \pi / \lambda$. Various lattice structures can be realized, depending on relative intensities of the beams, and we consider the regime where the hexagonal lattice is formed [see Figs. 1(b) and 1(c)]. The single-beam lattice depths $V_{\bar{X}}, V_{X}$, and $V_{Y}$ are set to be the same for two different layers. A gap could be generated at the Dirac point by tuning the laser parameter $\theta$. We also take $\varphi_{1}=0, \varphi_{2}=\pi$ (the phase between two orthogonal primary lasers $X$ and $Y$ ), which forms $A$ and $B$ sublattices with the opposite energy offset for layer 1 and layer 2 . Besides the (a) AA-stacked
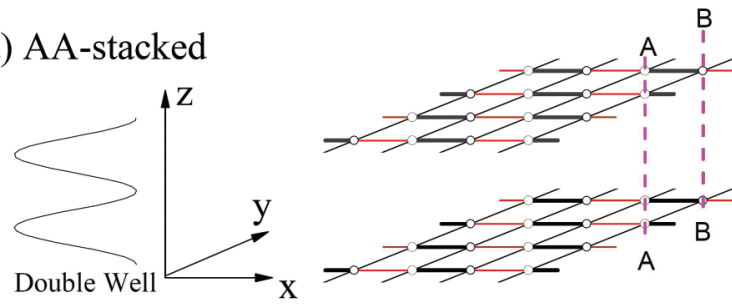

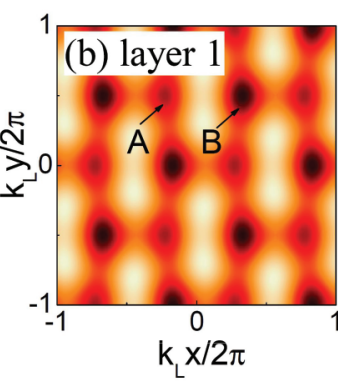

(d) layer $1 \& 2$

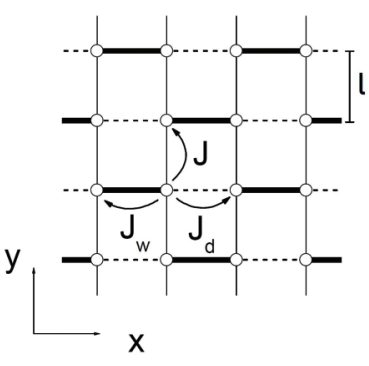

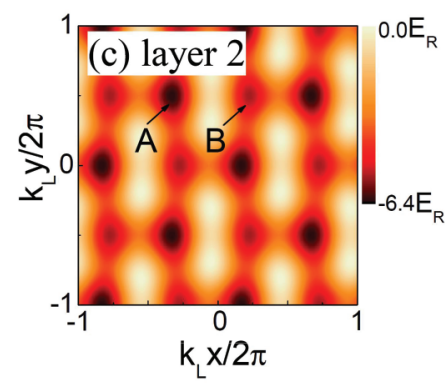

(e) Brillouin Zone

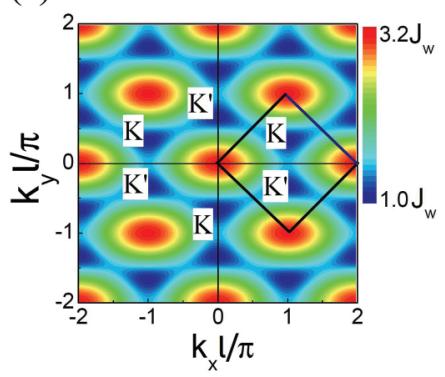

FIG. 1. (a) The proposed $A A$-stacked bilayer optical lattice, where the corresponding potential of layer 1 (b) and layer 2 (c) can be realized in the $x-y$ plane depending on the interference of the three laser beams. A sublattice offset could be generated in both layers under the condition, say, $\theta=0.5 \pi$ and the two layers are set with opposite on-site energy offset. An auxiliary double well is set along the $z$ direction for tuning the coupling between the two layers. Sketch of the tight-binding model is given (d) and topologically the quadratic lattice is equivalent to the hexagonal lattice. (e) Within the first Brillouin zone, there are two Dirac points, namely, $\mathbf{K}$ and $\mathbf{K}^{\prime}$.

2D optical lattice potential, we also include a double-well trap along the $z$ direction for tuning the interlayer coupling, which is necessary for the tomography of the final GMP state [see Sec. IV]. Below we first consider a deep trap and hence the two layers are treated isolated. The depth of the potential in $x-y$ plane can also be tuned such that the localized Wannier functions decay very rapidly with distance. Under the tight-binding approximation, only the tunneling terms between nearest-neighboring sites in $x-y$ are considered and the corresponding tight-binding Hamiltonian is written as

$$
H=\sum_{\mu=1,2} \sum_{\langle i, j\rangle} J_{\langle i, j\rangle, \mu}\left(a_{i, \mu}^{\dagger} b_{j, \mu}+\text { H.c. }\right)+\Delta_{\langle i, j\rangle, \mu} n_{\langle i, j\rangle, \mu},
$$

where $\langle i, j\rangle$ denotes the nearest-neighboring sites in each unit cell. $a_{i, \mu}$ and $b_{i, \mu}$ represent the boson annihilation operator at site $i$ for the sublattices $A$ and $B$ in layer $\mu$, respectively. The tunneling rates $J_{\langle i, j\rangle, \mu}$, in general, depend on the 
tunneling directions in an anisotropic hexagonal lattice and we denote them as $J, J_{w}, J_{d}$ corresponding to the three different directions [see Figs. 1(d) and 1(e)]. $\Delta_{\langle i, j\rangle, \mu}$ denotes the onsite energy in layer $\mu$ and we set $\Delta_{\mu, A}=-\Delta_{\mu, B}=\Delta_{\mu}$. The positions of the sites in sublattice $A$ can be expressed as $\mathbf{A}_{i}=$ $m_{1} \mathbf{a}_{1}+m_{2} \mathbf{a}_{2}$, where $m_{1}, m_{2}$ are integers and the basis vector $\mathbf{a}_{1}=(l, l)$ and $\mathbf{a}_{2}=(l,-l)$. The sites in the sublattice $B$ can then be generated by a shift $\mathbf{B}_{j}=\mathbf{A}_{j}+\boldsymbol{\delta}_{i}$, where the vector $\boldsymbol{\delta}_{i}$ could be an arbitrary one from four possible shift vectors $\boldsymbol{\delta}_{1}=(l, 0), \boldsymbol{\delta}_{2}=(-l, 0), \boldsymbol{\delta}_{3}=(0, l)$, and $\boldsymbol{\delta}_{4}=(0,-l)$ [see Figs. 1(d) and 1(e)]. Hereafter, we set the lattice spacing $l=1$ for simplicity.

With a Fourier transform $a_{j, \mu}=\frac{1}{\sqrt{N}} \sum_{\mathbf{k}, \mu} \exp \left(i \mathbf{k} \cdot \mathbf{A}_{j}\right) a_{\mathbf{k}, \mu}$ and $b_{j, \mu}=\frac{1}{\sqrt{N}} \sum_{\mathbf{k}, \mu} \exp \left(i \mathbf{k} \cdot \mathbf{B}_{j}\right) b_{\mathbf{k}, \mu}$, where $N$ is the number of the unit cell, the Hamiltonian simplifies to

$$
\begin{aligned}
H & =\sum_{\mathbf{k}, \mu}\left(\begin{array}{ll}
a_{\mathbf{k}, \mu}^{\dagger} & b_{\mathbf{k}, \mu}^{\dagger}
\end{array}\right) \mathcal{H}_{\mu}(\mathbf{k})\left(\begin{array}{l}
a_{\mathbf{k}, \mu} \\
b_{\mathbf{k}, \mu}
\end{array}\right), \\
\mathcal{H}_{\mu}(\mathbf{k}) & =\left[\begin{array}{cc}
\Delta_{\mu} & \epsilon_{\mathbf{k}} \\
\epsilon_{\mathbf{k}}^{*} & -\Delta_{\mu}
\end{array}\right],
\end{aligned}
$$

where $\epsilon_{\mathbf{k}} \equiv 2 J \cos k_{y}+J_{w} e^{-i k_{x}}+J_{d} e^{i k_{x}}$ and $\mathcal{H}_{\mu}(\mathbf{k})$ is the Bloch Hamiltonian. The energy eigenvalues are given by $E_{\mu}(\mathbf{k})= \pm \sqrt{\epsilon_{\mathbf{k}}^{2}+\Delta_{\mu}^{2}}$, which has the expression

$$
\begin{aligned}
E_{\mu}^{2}(\mathbf{k})= & \left(J_{d}-J_{w}\right)^{2}+4 J^{2} \cos ^{2}\left(k_{y}\right)+4 J_{d} J_{w} \cos ^{2}\left(k_{x}\right) \\
& +4 J\left(J_{d}+J_{w}\right) \cos \left(k_{x}\right) \cos \left(k_{y}\right)+\Delta_{\mu}^{2} .
\end{aligned}
$$

Within the regime $J_{w}<2 J-J_{d}$, the energy spectra exhibit linear dispersion at two Dirac points $\mathbf{K}, \mathbf{K}^{\prime} \equiv$ $\left(0, \pm \arccos \frac{J_{d}+J_{w}}{-2 J}\right)$. The increase of the tunneling strength $J_{w}$ leads to a merge of Dirac points in pairs along the $k_{y}$ direction. When $J_{w}>2 J-J_{d}$, the energy spectrum is linear in $k_{x}$, while it becomes quadratic in $k_{y}$. Since we need those two Majorana fermions (with opposite masses) to form a GMP, we consider the case of $J<2 J-J_{d}$. We plot the dispersion relation in the first Brilloiun zone [see Fig. 1(e)] with parameters $J_{d}=0$, $J_{w}=1, J / J_{w}=\sqrt{2}$, and $\Delta_{\mu} / J_{w}= \pm 1$.

By expanding the momentum as $\mathbf{k}=\mathbf{K}+\mathbf{q}$ around the high-symmetry point $\mathbf{K}$, where $\mathbf{q}=\left(q_{x}, q_{y}, q_{z}\right)$ is the displacement vector measured from the $\mathbf{K}$ point in the reciprocal space. One can obtain the dispersion relation Eq. (6) as

$$
E_{\mu}(\mathbf{q})= \pm \sqrt{v_{x}^{2} q_{x}^{2}+v_{y}^{2} q_{y}^{2}+\Delta_{\mu}^{2}}
$$

where $v_{x}=J_{d}-J_{w}, \quad v_{y}=\sqrt{4 J^{2}-\left(J_{d}+J_{w}\right)^{2}}$. The wave function for the quasiparticles satisfies the Dirac equation $i \hbar \partial_{t} \psi_{\mu}=\mathcal{H}_{\mu}^{\prime} \psi_{\mu}$, where the relativistic Hamiltonian is derived by linearizing the Bloch Hamiltonian at the $\mathbf{K}$ point. Up to a rotation in the $x y$ plane, i.e., $\left(\sigma_{x}, \sigma_{y}\right) \rightarrow\left(-\sigma_{y},-\sigma_{x}\right)$, the effective Hamiltonian can be written as

$$
\mathcal{H}_{\mu}^{\prime}(\mathbf{q})=v_{x} q_{x} \sigma_{x}+v_{y} q_{y} \sigma_{y}+\Delta_{\mu} \sigma_{z} .
$$

Due to the opposite on-site energy offset in layers 1 and 2, the effective mass $\Delta_{1}=-\Delta_{2}$. By equating $\mathcal{H}_{\mu}^{\prime}$ with $h_{\mu}$ in Eq. (2) we arrive at

$$
\begin{aligned}
& h_{1}(\mathbf{q})=v_{x} q_{x} \sigma_{x}+v_{y} q_{y} \sigma_{y}+M \sigma_{z}, \\
& h_{2}(\mathbf{q})=v_{x} q_{x} \sigma_{x}+v_{y} q_{y} \sigma_{y}-M \sigma_{z},
\end{aligned}
$$

where $M \equiv \Delta_{1}$. Correspondingly we write $\psi_{1}$ and $\psi_{2}$ as twocomponent Dirac wave functions for positive- and negativemass Dirac equations, respectively. The total wave function $\psi_{D}=\left(\psi_{1}, \psi_{2}\right)^{T}$ thus is a four-component one and the total Hamiltonian of bilayer hexagonal lattice exhibits a blockdiagonal form. Using $\left(\tau_{0}, \tau\right)$ to denote the Pauli matrices in the layer space, the full Dirac Hamiltonian can then be written as

$$
\mathcal{H}_{D}=\left(v_{x} q_{x} \tau_{0} \otimes \sigma_{x}+v_{y} q_{y} \tau_{0} \otimes \sigma_{y}\right)+M \tau_{z} \otimes \sigma_{z} .
$$

Up to now the dynamics of the GMP can be simulated with the Dirac Hamiltonian shown in Eq. (10) as well as the corresponding Dirac state. To derive the final GMP state, we introduce the interlayer coupling for the tomography of the GMP states, which will be shown in Sec. IV. Since the two layers are $A A$ stacked, we write the interaction Hamiltonian as $H_{\text {int }}=\sum_{i} J_{\perp} e^{-i \varphi_{\perp}}\left(a_{i, 1}^{\dagger} a_{i, 2}+b_{i, 1}^{\dagger} b_{i, 2}\right)+$ H.c., where $J_{\perp} e^{i \varphi_{\perp}}$ denotes the tunneling rate in the $z$ direction that can be realized by, say, laser-assisted transition [48,56,57]. In the reciprocal space, it is written as

$$
\mathcal{H}_{\text {int }}=J_{\perp} \cos \varphi_{\perp} \tau_{x} \otimes \sigma_{0}+J_{\perp} \sin \varphi_{\perp} \tau_{y} \otimes \sigma_{0},
$$

where $\sigma_{0}$ denotes the identity matrix in the sublattice space. In the following section, we will show that such interlayer coupling is also feasible for phase imprinting of the GMP state.

\section{MAJORANA DYNAMICS}

To verify the simulation of the GME, we investigate the observables pseudoenergy, orthogonality, and fidelity, which have been treated as the signatures of Majorana dynamics [4-7]. We start with a simple theoretical analysis on the free evolution of the GMP in the rest frame. Under such circumstances, the dynamics of Majorana and Dirac particles show the most difference [6] and the Majorana equation becomes a very compact form as

$$
\partial_{t} \Psi=-M \sigma_{y} \Psi^{*} .
$$

Taking complex conjugates on both sides of the equation, we obtain $\partial_{t} \Psi^{*}=M \sigma_{y} \Psi$ and $\partial_{t}^{2} \Psi=-M^{2} \Psi$. Through simple calculation, the general solution of Eq. (12) can be obtained as

$$
\Psi(t)=\cos (M t) \Psi(0)-\sin (M t) \sigma_{y} \Psi^{*}(0) .
$$

Pseudoenergy, orthogonality, and fidelity. In the rest frame, the Dirac Hamiltonian is reduced to $h_{\mu}=\Delta_{\mu} \sigma_{z}$ and the operator $\sigma_{z}$ or pseudoenergy corresponds to a conserved quantity. In contrast, the GMP consisting of two coherent Majorana fermions prohibits the conservation of the pseudoenergy. By inserting the wave function in Eq. (13) into the definition, the pseudoenergy is obtained as

$$
\begin{aligned}
\left\langle\sigma_{z}(t)\right\rangle= & \left\langle\Psi(t)\left|\sigma_{z}\right| \Psi(t)\right\rangle \\
= & \cos (2 M t) \Psi^{\dagger}(0) \sigma_{z} \Psi(0) \\
& +\sin (2 M t) \operatorname{Im}\left[\Psi^{T}(0) \sigma_{x} \Psi(0)\right],
\end{aligned}
$$

where $\operatorname{Im}[c]$ denotes the imaginary part of complex number $c$. For the same reason, the orthogonality of two initial states will not be maintained during the evolution and it is given by

$$
\left|\left\langle\Psi(t) \mid \Psi_{\perp}(t)\right\rangle\right|^{2}=\sin ^{2}(2 M t)\left[\operatorname{Im}\left[\Psi^{T}(0) \sigma_{y} \Psi_{\perp}(0)\right]\right]^{2},
$$


where $\Psi_{\perp}(t)$ represents the time-evolved state of the initial one $\Psi_{\perp}(0)$, which is orthogonal to the initial state $\Psi(0)$. In a similar way, the fidelity is defined as

$$
\left|\left\langle\Psi(t) \mid \Psi_{\theta}(t)\right\rangle\right|^{2}=\left|\cos ^{2}(\theta)+\sin ^{2}(\theta) \cos ^{2}(2 M t)\right|^{2},
$$

where $\Psi_{\theta}$ denotes the wave function that evolves from initial state $\Psi(0) e^{i \theta}$ with $\theta$ being a constant. The fidelity oscillation arises due to the fact that the GME has no global U(1) symmetry, which is completely different from Dirac/Weyl dynamics [3]. The GMP states $\Psi$ and $\Psi e^{i \theta}$ are in general two different states, which can be explicitly seen by decomposing them into corresponding Majorana fermions. Taking $\Psi=\psi_{1}+i \psi_{2}$, where $\psi_{1}$ and $\psi_{2}$ satisfy the Majorana fermion condition, then we have $\Psi e^{i \theta}=\left(\cos \theta \psi_{1}-\sin \theta \psi_{2}\right)+i\left(\sin \theta \psi_{1}+\cos \theta \psi_{2}\right)$ or

$$
\Psi \rightarrow \Psi_{\theta}=\left[\begin{array}{cc}
\cos \theta & -\sin \theta \\
\sin \theta & \cos \theta
\end{array}\right]\left(\begin{array}{l}
\psi_{1} \\
\psi_{2}
\end{array}\right)
$$

where each matrix element is implicitly multiplied with a $2 \times$ 2 identity matrix. In other words, a U(1) transformation of the GMP state is manifested as a $\mathrm{SO}(2)$ rotation of corresponding two Majorana fermions. Writing the rotation matrix as $U_{R}=$ $e^{-i \tau_{y} \otimes \sigma_{0} \theta}$, one can recognize such operation as an evolution matrix with Hamiltonian given by Eq. (11), which enables phase imprinting of the GMP state in experiments by tuning the interlayer coupling.

In the cold-atom system, we consider a cold atomic ensemble trapped in a 2D harmonic well for experimental investigation of the dynamics and observables. We take the initial GMP state in the form

$$
\Psi=\frac{1}{\sqrt{2 \pi} L} e^{-\left(x^{2}+y^{2}\right) / 4 L^{2}+i \mathbf{K} \cdot x} \Phi,
$$

where $\Phi$ denotes the spinor part of the wave function and $L$ represents the width of the initial Gaussian profile. The factor $\exp (i \mathbf{K} \cdot x)$ is included which renders the GMP state localized at the Dirac point $\mathbf{K}$ in the reciprocal space. The evolution therefore is governed by the effective Hamiltonian given in Eq. (10). For large width $L$, the evolution of the spinor wave function is mainly assumed by the mass term. The contribution from the momentumdependent terms can be neglected and hence the previous results in the rest frame can be applied. For a numerical demonstration, we have computed the evolutionary process of a GMP by solving the Schrödinger's equation with Hamiltonian Eq. (10). For a general view on the GMP state evolution, we take three typical initial spinors $\left[\Phi_{x}=\right.$ $(1 / \sqrt{2})(1,1)^{T}, \Phi_{y}=(1 / \sqrt{2})(1, i)^{T}$, and $\Phi_{z}=(1,0)^{T}$, by which any spinor can be constructed] into consideration. By decomposing the initial state into two Majorana fermion states, the corresponding Dirac spinors are $\phi_{x, 1}=(0,0)^{T}$, $\phi_{x, 2}=(1 / \sqrt{2})(-i,-i)^{T}, \phi_{y, 1}=(1 / 2)(1+i, i-1)^{T}, \phi_{y, 2}=$ $-(1 / 2)(1+i, i-1)^{T}, \phi_{z, 1}=(1 / \sqrt{2})(1,-1)^{T}$, and $\phi_{z, 2}=$ $(1 / \sqrt{2})(-i,-i)^{T}$, where $\phi_{x, y, z, 1}\left(\phi_{x, y, z, 2}\right)$ corresponds to the Dirac quasiparticle in layer 1 (2). For the pseudoenergy and orthogonality with initial spinor given by the three typical
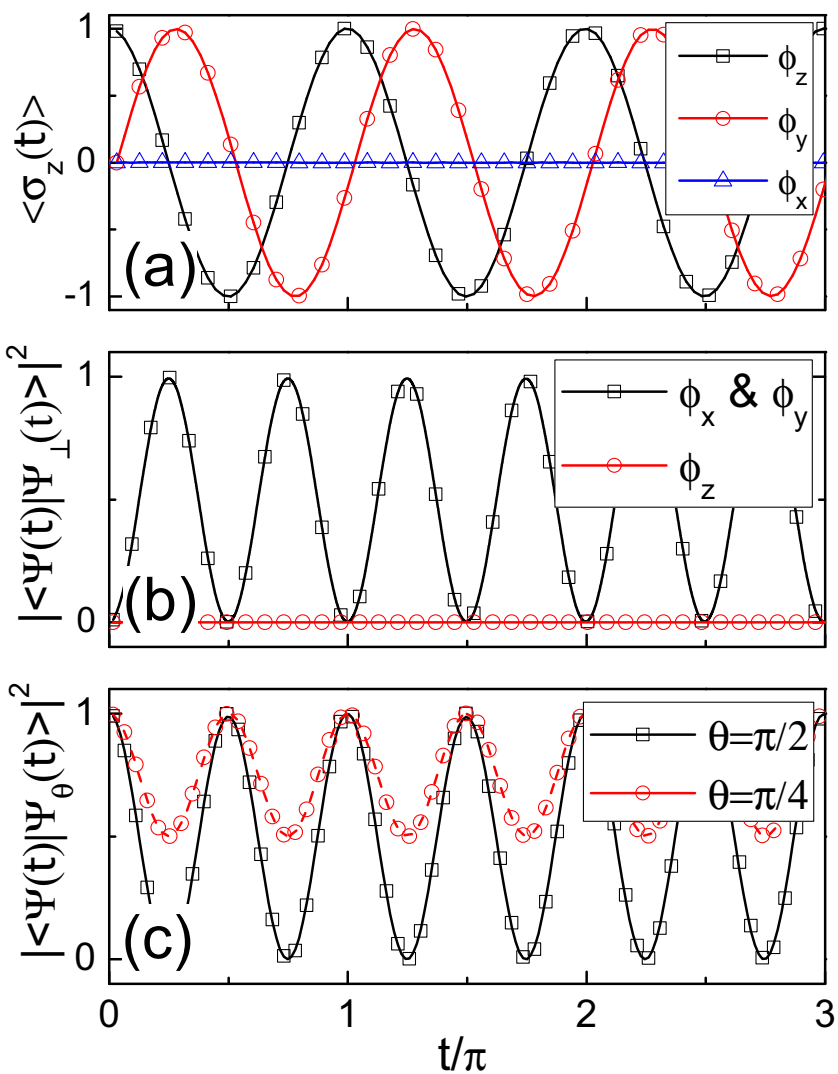

FIG. 2. Pseudoenergy (a), orthogonality (b), and fidelity (c) of a GMP with the three typical initial spinors $\left(\Phi_{x}, \Phi_{y}\right.$, and $\left.\Phi_{z}\right)$. The fidelity oscillation in (c) is independent of the initial spinor wave function while it depends on the phase difference $\theta$ of the initial GMP states. The lines (symbols) represent the analytical (numerical) results, which shows good agreement. In computation, the width of initial wave function $L=10$ and the effective mass $M=1$. The time $t$ is in units of $1 / J_{w}$.

spinors, we have

$$
\left\langle\sigma_{z}(t)\right\rangle=\left\{\begin{array}{lll}
0 & \text { for } & \Phi_{x} \\
\sin (2 M t) & \text { for } & \Phi_{y} \\
\cos (2 M t) & \text { for } & \Phi_{z}
\end{array}\right.
$$

and

$$
\left|\left\langle\Psi(t) \mid \Psi_{\perp}(t)\right\rangle\right|^{2}=\left\{\begin{array}{lll}
\sin ^{2}(2 M t) & \text { for } & \Phi_{x} \\
\sin ^{2}(2 M t) & \text { for } & \Phi_{y} \\
0 & \text { for } & \Phi_{z}
\end{array}\right.
$$

Both analytic (lines) and numerical (symbols) results are plotted in Fig. 2, which shows good agreements. The fidelity oscillation does not rely on the initial spinor and the periodic oscillation always occurs with a nonzero global phase factor. It means that the fidelity is a good quantity to distinguish the Majorana from Dirac/Weyl dynamics [2,28,36].

Majorana Zitterbewegung. Apart from the observables relevant to the spinor wave function, we proceed to investigate the spatial part of the wave function, which shows Zitterbewegung (ZB) [5,58]. The $\mathrm{ZB}$ effect historically was first derived by solving the Dirac equation, which exhibits an oscillatory behavior in the particle's motion. For the GMP, the 
decomposition in Eq. (2) enables an analytical investigation of the corresponding analog in the GME. The Dirac ZB effect in experiments can be examined by monitoring the center of mass of wave packets [16]. Similarly, for the GMP state we calculate the evolution of the expectation values $\overline{\mathbf{r}}(t)=$ $\langle\Psi(t)|\hat{\mathbf{r}}| \Psi(t)\rangle$. By its definition, we have

$$
\begin{aligned}
\overline{\mathbf{r}}(t)= & \langle\Psi(t)|\hat{\mathbf{r}}| \Psi(t)\rangle \\
= & \frac{1}{2}\left\langle\psi_{1}(t)|\hat{\mathbf{r}}| \psi_{1}(t)\right\rangle+\frac{1}{2}\left\langle\psi_{2}(t)|\hat{\mathbf{r}}| \psi_{2}(t)\right\rangle \\
& +\frac{i}{2}\left\langle\psi_{1}(t)|\hat{\mathbf{r}}| \psi_{2}(t)\right\rangle-\frac{i}{2}\left\langle\psi_{2}(t)|\hat{\mathbf{r}}| \psi_{1}(t)\right\rangle .
\end{aligned}
$$

The first two terms are the usual Dirac ZB associated to each Majorana fermion while the last two terms are the $\mathrm{ZB}$ resulted from the transition between the two Majorana fermion states. An analytical derivation can be done in the Heisenberg picture. Defining the operators

$$
\hat{\mathbf{r}}_{1,2}(t)=e^{i h_{1,2} t} \hat{\mathbf{r}}(0) e^{-i h_{1,2} t}, \quad \hat{\mathbf{r}}_{12}(t)=e^{i h_{1} t} \hat{\mathbf{r}}(0) e^{-i h_{2} t},
$$

we can rewrite $\overline{\mathbf{r}}(t)$ as

$$
\begin{aligned}
\overline{\mathbf{r}}(t)= & \frac{1}{2}\left\langle\psi_{1}(0)\left|\hat{\mathbf{r}}_{1}(t)\right| \psi_{1}(0)\right\rangle+\frac{1}{2}\left\langle\psi_{2}(0)\left|\hat{\mathbf{r}}_{2}(t)\right| \psi_{2}(0)\right\rangle \\
& +\frac{i}{2}\left\langle\psi_{1}(0)\left|\hat{\mathbf{r}}_{12}(t)\right| \psi_{2}(0)\right\rangle-\frac{i}{2}\left\langle\psi_{2}(0)\left|\hat{\mathbf{r}}_{12}^{\dagger}(t)\right| \psi_{1}(0)\right\rangle .
\end{aligned}
$$

For $\hat{\mathbf{r}}_{12}$, we obtain

$$
\begin{aligned}
\hat{\mathbf{r}}_{12}(t)= & {\left[1+i M \sigma_{z} \frac{\sin 2 E t}{E}\right.} \\
& \left.+i 2 M \frac{\sin ^{2} E t}{E^{2}}\left(v_{x} p_{x} \sigma_{y}-v_{y} p_{y} \sigma_{x}\right)\right] \hat{\mathbf{r}}_{2}(t),
\end{aligned}
$$

where $E=\sqrt{v_{x}^{2} q_{x}^{2}+v_{y}^{2} q_{y}^{2}+M^{2}} \cdot \hat{\mathbf{r}}_{1,2}(t)$ can be solved by using the anticommutation properties of the Pauli matrices and one finds

$$
\begin{aligned}
\hat{x}_{1,2}(t)= & \hat{x}(0)+v_{x}^{2} h_{1,2}^{-1} q_{x} t \\
& -\frac{i v_{x}}{2} h_{1,2}^{-1}\left(e^{i 2 h_{1,2} t}-1\right)\left(\sigma_{x}-v_{x} h_{1,2}^{-1} q_{x}\right), \\
\hat{y}_{1,2}(t)= & \hat{y}(0)+v_{y}^{2} h_{1,2}^{-1} q_{y} t \\
& -\frac{i v_{y}}{2} h_{1,2}^{-1}\left(e^{i 2 h_{1,2} t}-1\right)\left(\sigma_{y}-v_{y} h_{1,2}^{-1} q_{y}\right) .
\end{aligned}
$$

By substituting Eqs. (22)-(25) into Eq. (21), an exact formula can be derived for $\mathbf{\mathbf { r }}(t)$ and one can find that the last two terms in Eq. (21) are exactly canceled. For the wave packet given in Eq. (18), however, we could simplify the calculation by taking $\mathbf{q} \rightarrow 0$, which corresponds to the large width limit of the wave packet, i.e., $L \rightarrow \infty$. Then we obtain

$$
\hat{\mathbf{r}}_{12}(t)=e^{i 2 M \sigma_{z} t} \hat{\mathbf{r}}_{2}(t),
$$

and

$$
\begin{aligned}
& \hat{x}_{1,2}(t)=\hat{x}_{1,2}(0) \pm \frac{v_{x}}{2 M} \sigma_{y}(\cos 2 M t-1)+\frac{v_{x}}{2 M} \sigma_{x} \sin 2 M t, \\
& \hat{y}_{1,2}(t)=\hat{y}_{1,2}(0) \mp \frac{v_{y}}{2 M} \sigma_{x}(\cos 2 M t-1)+\frac{v_{y}}{2 M} \sigma_{y} \sin 2 M t .
\end{aligned}
$$
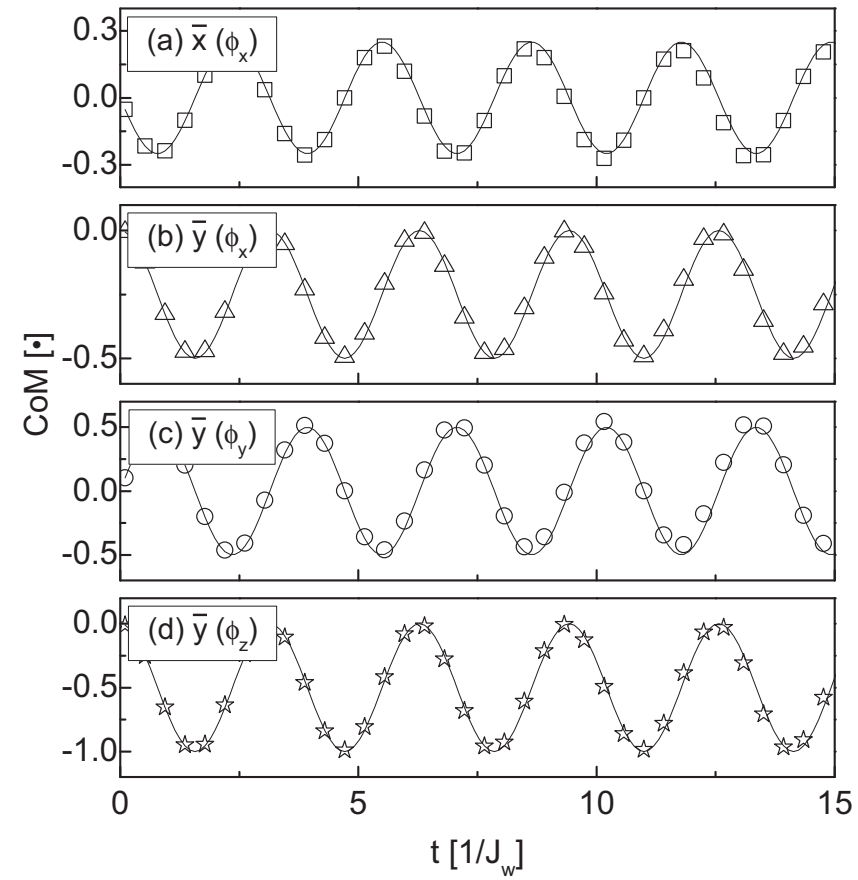

FIG. 3. Majorana Zitterbewegung for different initial spinors. The analytical (lines) and numerical (symbols) results of the center of mass (CoM) of a GMP with $L=10$ are plotted. (a) and (b) show the $\bar{x}$ and $\bar{y}$ for $\Phi_{x}$; (c) and (d) provide $\bar{y}$ for $\Phi_{y}$ and $\Phi_{z}$, respectively. Throughout, the effective mass $M / J_{w}=1$.

For the Majorana fermion condition, we further parametrize the two Majorana fermion states as $\psi_{1}(0)=1 / \sqrt{2}$ $\left(e^{i \phi_{1} / 2},-e^{-i \phi_{1} / 2}\right)^{T} \quad$ and $\quad \psi_{2}(0)=1 / \sqrt{2}\left(e^{i \phi_{2} / 2},-e^{-i \phi_{2} / 2}\right)^{T}$. The final result is just the addition of the two Majorana fermion's ZB oscillation, which is given by

$$
\begin{aligned}
& \bar{x}(t)=-\frac{v_{x}}{4 M}\left[\sin \left(2 M t-\phi_{1}\right)+\sin \left(2 M t+\phi_{2}\right)\right], \\
& \bar{y}(t)=\frac{v_{y}}{4 M}\left[\cos \left(2 M t-\phi_{1}\right)-\cos \left(2 M t+\phi_{2}\right)\right],
\end{aligned}
$$

where we have neglected constant terms, which correspond to the initial position of the wave packet. For the three initial GMP spinors, we obtain

$$
\bar{x}=\frac{v_{x}}{4 M} \sin 2 M t, \quad \bar{y}=\frac{v_{y}}{4 M} \cos 2 M t
$$

for $\Phi_{x}$,

$$
\bar{x}=0, \quad \bar{y}=\frac{v_{y}}{2 M} \sin 2 M t
$$

for $\Phi_{y}$, and

$$
\bar{x}=0, \quad \bar{y}=\frac{v_{y}}{2 M} \cos 2 M t
$$

for $\Phi_{z}$. A numerical comparison has been given in Fig. 3. For a broad wave packet, the numerical results agree well with the analytical ones given above. For a smaller width, damping behavior and drift velocity shall be exhibited as discussed in Refs. [58-60]. 


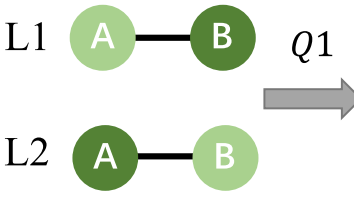

(a)

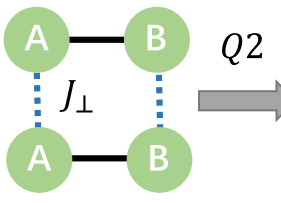

(b)

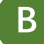

(c)
FIG. 4. Quench steps for yielding the density matrix of the Majorana state. (a) Initially the layers are decoupled and $A B$ sublattices are energetically separated. In the step $Q 1$, interlayer coupling is turned on while the energy difference between sublattices is turned off at the same time. In stage (b), the Majorana state is prepared in the first layer. The state tomography is implemented in stage (c), in which the system is quenched such that sublattices are energetically separated by $\hbar \omega$ and all the tunnelings are suppressed. The final time-of-flight measurement is implemented only on the first layer.

\section{EXPERIMENTAL PROCEDURE AND MEASUREMENT}

In previous sections, we have proposed a bilayer optical lattice for simulation of the Dirac equation and investigated featured observables in the Majorana dynamics. To simulate the evolution of a GMP state, we need first to decompose it into the two Majorana fermion states, say, $\psi_{1}(0)$ and $\psi_{2}(0)$. Then prepare the initial state as $\psi_{D}(0)=\left[\psi_{1}(0), \psi_{2}(0)\right]^{T}$ and time evolve the state in the bilayer system described by the Dirac Hamiltonian, Eq. (10). The final step is to evaluate the observables with the final state $\psi_{D}(t)=\left[\psi_{1}(t), \psi_{2}(t)\right]^{T}$ or the GMP state $\Psi=\psi_{1}(t)+i \psi_{2}(t)$, which are required to be experimentally measured. Here we propose two quench processes to complete the final experimental measurements, which are pictured in Fig. 4. The first quench is to transform the Dirac state into the GMP state since a Dirac state rather than a GMP state is obtained in the simulation. The second is to realize the state tomography of the derived GMP state.

In the first quench process, the interlayer coupling is switched on while at the same time the energy offset between the $A B$ sublattices in each layer is turned off. According to Eqs. (10) and (11), the system is then described by the following effective Hamiltonian:

$$
H_{Q 1}=\left[\begin{array}{cc}
v_{x} p_{x} \sigma_{x}+v_{y} p_{y} \sigma_{y} & J_{\perp} \sigma_{0} \\
J_{\perp} \sigma_{0} & v_{x} p_{x} \sigma_{x}+v_{y} p_{y} \sigma_{y}
\end{array}\right],
$$

where $J_{\perp}$ denotes the interlayer hopping strength. Under the condition $J, J_{w} \ll J_{\perp}$, we could neglect the block diagonal term. For a suitable duration of evolution with the simplified Hamiltonian, one can obtain the following evolution matrix:

$$
U_{Q 1}=\frac{1}{\sqrt{2}}\left[\begin{array}{cc}
\sigma_{0} & i \sigma_{0} \\
i \sigma_{0} & \sigma_{0}
\end{array}\right],
$$

which renders the Dirac states $\left|\psi_{1,2}\right\rangle$ of the previously decoupled layers as

$$
\left|\psi_{1}\right\rangle \rightarrow\left|\psi_{1}\right\rangle+i\left|\psi_{2}\right\rangle, \quad\left|\psi_{2}\right\rangle \rightarrow i\left|\psi_{1}\right\rangle+\left|\psi_{2}\right\rangle .
$$

Through this procedure, the GMP state is prepared in the first layer and we proceed to the next quench process for the state tomography.
The state tomography of the GMP state is implemented with the quench that all the tunneling is suppressed while the sublattices are energetically separated [55]. Supposing the energy offset between the $A$ and $B$ sublattices of the quenched system is $\omega$, then the Hamiltonian of the post-quenched system is characterized by $H_{Q 2}=\omega \sigma_{z} / 2$, which generates a rotation around the $z$ axis of the Bloch sphere. The time evolution conserves the momentum and mainly modifies the spinor part of the wave function. Therefore the spatial wave function of the final state is taken to be Gaussian. Therefore we write the final GME state in the reciprocal space in the form

$$
|\Psi(t)\rangle=\frac{L}{\sqrt{\pi}} e^{-(1 / 2) \mathbf{q}^{2} L^{2}}\left(\begin{array}{c}
\sin \frac{\beta}{2} \\
\cos \frac{\beta}{2} e^{i \gamma}
\end{array}\right) .
$$

By holding the gas in the quenched lattice for time interval $t_{\mathrm{h}}$, the momentum distribution of the gases is given by

$$
n\left(\mathbf{k}, t_{\mathrm{h}}\right)=f(\mathbf{k}) \frac{L^{2}}{\pi} e^{-(\mathbf{k}-\mathbf{K})^{2} L^{2}}\left[1+\sin \beta \cos \left(\gamma+\omega t_{\mathrm{h}}\right)\right],
$$

where $f(\mathbf{k})$ denotes the momentum distribution of the Wannier function associated to the lattice sites and we have used the relation $\mathbf{q}=\mathbf{k}-\mathbf{K}$. By measuring the $n\left(\mathbf{k}, t_{\mathrm{h}}\right)$ for different hold time $t_{\mathrm{h}}$, both $\beta(t)$ and $\gamma(t)$ can be obtained, yielding the desired density matrix of the GMP state.

Given the density matrix of the GME state, the proposed observables can be readily obtained. The pseudoenergy has the simple form $\left\langle\sigma_{z}\right\rangle=-\cos \beta(t)$ while the other two quantities can be constructed from the overlap of the time-evolved states starting from different initial states. For example, the orthogonality can be rewritten with the time-evolved density matrices of the two initially orthogonal states

$$
\left|\left\langle\Psi(t) \mid \Psi_{\perp}(t)\right\rangle\right|^{2}=\operatorname{Tr}\left(\rho \rho_{\perp}\right),
$$

where $\rho(t) \equiv|\Psi(t)\rangle\langle\Psi(t)|$ and $\rho_{\perp}(t) \equiv\left|\Psi_{\perp}(t)\right\rangle\left\langle\Psi_{\perp}(t)\right|$ and corresponding initial states satisfy the condition $\left\langle\Psi(0) \mid \Psi_{\perp}(0)\right\rangle=0$.

\section{CONCLUSION}

In summary, we have proposed an $A A$-stacked bilayer hexagonal lattice to simulate the general Majorana equation and observe the dynamics with an atomic gas in a cold-atom system. The evolution generated by the general Majorana equation is nonunitary in general. However, the general Majorana equation can be cast into two Dirac equations with opposite masses, which enables the experimental simulation of the nonunitary evolution. In the context of a cold-atom system, we explored the possibility of realizing the Dirac equations with a bilayer optical lattice. In contrast to the Dirac/Weyl case [2,28,36,61], there exist the oscillations of pseudoenergy, orthogonality, and fidelity, as well as the intriguing Majorana Zitterbewegung in the evolutionary process. The evolution can be implemented by holding an atomic ensemble in the bilayer lattice. Through absorption imaging and quantum-state tomography, the density distribution and the density matrices of the GMP state can be detected. The necessary techniques for experiments are widely conducted by several groups $[54,57,62]$. The oscillating frequency of the observable quantities can be controlled in a detectable range 
and hence the observation of the aforementioned intriguing phenomena are expectable in cold atomic experiments [63]. We hope this work will not only contribute to the fundamental study of relativistic dynamics, but also promote the progress of quantum simulators [64].

\section{ACKNOWLEDGMENTS}

We are grateful to J.-N. Zhang and S.-C. Zhang for useful discussions. X.S. thanks Fu-dong Wang and Zhigang $\mathrm{Wu}$ for helpful discussions. This work was supported by the
National Key Research and Development Program of China (Grants No. 2016YFA0301803 and No. 2016YFA0302800), National Natural Science Foundation of China (Grants No. 11604103, No. 11704132, No. 11822403,91636218, and No. U1801661), the NSAF (Grant No. U1830111), the PCSIRT (Grant No. IRT1243), the Natural Science Foundation of Guangdong Province (Grants No. 2016A030313436, No. 2018A030313322, and No. 2018A0303130066), the KPST of Guangzhou (Grant No. 201804020055), China Postdoctoral Science Foundation (Grant No. 2018M633063), and the Startup Foundation of SCNU.
[1] E. Majorana, Nuovo Cimento 14, 171 (1937).

[2] B. Thaller, The Dirac Equation (Springer, New York, 1992).

[3] A. Zee, Quantum Field Theory in a Nutshell (Princeton University, Princeton, NJ, 2003).

[4] J. Casanova, C. Sabín, J. León, I. L. Egusquiza, R. Gerritasma, C. F. Roos, J. J. García-Ripoll, and E. Solano, Phys. Rev. X 1, 021018 (2011).

[5] X. Zhang, Y. C. Shen, J. H. Zhang, J. Casanova, L. Lamata, E. Solano, M.-H. Yung, J.-N. Zhang, and K. Kim, Nat. Commun. 6, 7917 (2015).

[6] C. Noh, B. M. Rodríguez-Lara, and Dimitris G. Angelakis, Phys. Rev. A 87, 040102(R) (2013).

[7] R. Keil, C. Noh, A. Rai, S. Stützer, S. Nolte, Dimitris G. Angelakis, and A. Szameit, Optica 2, 454 (2015).

[8] D.-W. Zhang, Y.-Q. Zhu, Y. X. Zhao, H. Yan, and S.-L. Zhu, Adv. Phys. 67, 253 (2018).

[9] N. R. Cooper, J. Dalibard, and I. B. Spielman, Rev. Mod. Phys. 91, 015005 (2019).

[10] M. Lewenstein, A. Sanpera, V. Ahufinger, B. Damski, A. S. De, and U. Sen, Adv. Phys. 56, 243 (2007).

[11] J. Dalibard, F. Gerbier, G. Juzeliūnas, and P. Öhberg, Rev. Mod. Phys. 83, 1523 (2011).

[12] N. Goldman, G. Juzeliūnas, P. Öhberg, and I. B. Spielman, Rep. Prog. Phys. 77, 126401 (2014).

[13] S.-L. Zhu, B.-G. Wang, and L.-M. Duan, Phys. Rev. Lett. 98, 260402 (2007).

[14] L.-B. Shao, S.-L. Zhu, L. Sheng, D.-Y. Xing, and Z. D. Wang, Phys. Rev. Lett. 101, 246810 (2008).

[15] D.-W. Zhang, Z. D. Wang, and S.-L. Zhu, Front. Phys. 7, 31 (2012).

[16] L. J. LeBlanc, M. C. Beeler, K. Jiménez-García, A. R. Perry, S. Sugawa, R. A. Williams, and I. B. Spielman, New J. Phys. 15, 073011 (2013).

[17] P. Windpassinger and K. Sengstock, Rep. Prog. Phys. 76, 086401 (2013).

[18] M. I. Katsnelson, K. S. Novoselov, and A. K. Geim, Nat. Phys. 2, 620 (2006).

[19] N. Stander, B. Huard, and D. Goldhaber-Gordon, Phys. Rev. Lett. 102, 026807 (2009).

[20] S.-L. Zhu, D.-W. Zhang, and Z. D. Wang, Phys. Rev. Lett. 102, 210403 (2009).

[21] T. Salger, C. Grossert, S. Kling, and M. Weitz, Phys. Rev. Lett. 107, 240401 (2011).

[22] I. I. Rajbi, Z. Phys. 49, 507 (1928).

[23] J. W. McClure, Phys. Rev. 104, 666 (1956).
[24] G. Li and E. Y. Andrei, Nat. Phys. 3, 623 (2007).

[25] S.-L. Zhu, L.-B. Shao, Z. D. Wang, and L.-M. Duan, Phys. Rev. Lett. 106, 100404 (2011).

[26] A. H. Castro Neto, F. Guinea, N. M. R. Peres, K. S. Novoselov, and A. K. Geim, Rev. Mod. Phys. 81, 109 (2009).

[27] L. Tarruell, D. Greif, T. Uehlinger, G. Jotzu, and T. Esslinger, Nature (London) 483, 302 (2012).

[28] Z. Li, H. Cao, and L.-B. Fu, Phys. Rev. A 91, 023623 (2015).

[29] X. Wan, A. M. Turner, A. Vishwanath, and S. Y. Savrasov, Phys. Rev. B 83, 205101 (2011).

[30] S.-Y. Xu, I. Belopolski, N. Alidoust, M. Neupane, C. Zhang, R. Sankar, S. M. Huang, C. C. Lee, G. Chang, B. K. Wang, G. Bian, H. Zheng, D. S. Sanchez, F. Chou, H. Lin, S. Jia, and M. Z. Hasan, Science 347, 294 (2015).

[31] S.-Y. Xu, I. Belopolski, N. Alidoust, M. Neupane, G. Bian, C. Zhang, R. Sankar, G. Chang, Z. Yuan, C. C. Lee, S. M. Huang, H. Zheng, J. Ma, D. S. Sanchez, B. Wang, A. Bansil, F. Chou, Pavel P. Shibayev, H. Lin, S. Jia, and M. Z. Hasan, Science 349, 613 (2015)

[32] X. C. Huang, L. X. Zhao, Y. J. Long, P. P. Wang, D. Chen, Z. H Yang, H. Liang, M. Q. Xue, H. M. Weng, Z. Fang, X. Dai, and G. F. Chen, Phys. Rev. X 5, 031023 (2015).

[33] T. Dubcek, C. J. Kennedy, L. Lu, W. Ketterle, M. Soljacic, and H. Buljan, Phys. Rev. Lett. 114, 225301 (2015).

[34] D.-W. Zhang, S.-L. Zhu, and Z. D. Wang, Phys. Rev. A 92, 013632 (2015).

[35] W.-Y. He, S. Zhang, and K. T. Law, Phys. Rev. A 94, 013606 (2016).

[36] Z. Li, H.-Q. Wang, D.-W. Zhang, S.-L. Zhu, and D.-Y. Xing, Phys. Rev. A 94, 043617 (2016).

[37] V. Galitski and I. B. Spielman, Nature (London) 494, 49 (2013).

[38] H. Zhai, Rep. Prog. Phys. 78, 026001 (2015).

[39] Z. Wu, L. Zhang, W. Sun, X.-T. Xu, B.-Z. Wang, J.-C. Ji, Y. Deng, S. Chen, X.-J. Liu, and J.-W. Pan, Science 354, 83 (2016).

[40] X.-J. Liu, K. T. Law, and T. K. Ng, Phys. Rev. Lett. 112, 086401 (2014).

[41] K. Osterloh, M. Baig, L. Santos, P. Zoller, and M. Lewenstein, Phys. Rev. Lett. 95, 010403 (2005).

[42] J. Ruseckas, G. Juzeliūnas, P. Öhberg, and M. Fleischhauer, Phys. Rev. Lett. 95, 010404 (2005).

[43] S.-L. Zhu, H. Fu, C.-J. Wu, S.-C. Zhang, and L.-M. Duan, Phys. Rev. Lett. 97, 240401 (2006)

[44] X.-J. Liu, X. Liu, L. C. Kwek, and C. H. Oh, Phys. Rev. Lett. 98, 026602 (2007). 
[45] Y.-J. Lin, R. L. Compton, K. Jiménez-García, J. V. Porto, and I. B. Spielman, Nature (London) 462, 628 (2009).

[46] P. G. Harper, Proc. Phys. Soc. London, Sect. A 68, 874 (1955).

[47] D. R. Hofstadter, Phys. Rev. B 14, 2239 (1976).

[48] H. Miyake, G. A. Siviloglou, C. J. Kennedy, W. C. Burton, and W. Ketterle, Phys. Rev. Lett. 111, 185302 (2013).

[49] F. D. M. Haldane, Phys. Rev. Lett. 61, 2015 (1988).

[50] G. Jotzu, M. Messer, R. Desbuquois, M. Lebrat, T. Uehlinger, D. Greif, and T. Esslinger, Nature (London) 515, 237 (2014).

[51] B. K. Stuhl, H.-I. Lu, L. M. Aycock, D. Genkina, and I. B. Spielman, Science 349, 1514 (2015).

[52] X.-J. Liu, Z.-X. Liu, and M. Cheng, Phys. Rev. Lett. 110, 076401 (2013).

[53] S.-T. Wang, D.-L. Deng, and L.-M. Duan, Phys. Rev. Lett. 113, 033002 (2014).

[54] N. Fläschner, B. S. Rem, M. Tarnowski, D. Vogel, D.-S. Lühmann, K. Sengstock, and C. Weitenberg, Science 352, 1091 (2016).
[55] P. Hauke, M. Lewenstein, and A. Eckardt, Phys. Rev. Lett. 113, 045303 (2014).

[56] D. Jaksch and P. Zoller, New J. Phys. 5, 56 (2003).

[57] M. Aidelsburger, M. Atala, M. Lohse, J. T. Barreiro, B. Paredes, and I. Bloch, Phys. Rev. Lett. 111, 185301 (2013).

[58] W. Zawadzki and T. M. Rusin, J. Phys.: Condens. Matter 23, 143201 (2011).

[59] J. Y. Vaishnav and C. W. Clark, Phys. Rev. Lett. 100, 153002 (2008).

[60] M. Merkl, F. E. Zimmer, G. Juzeliūnas, and P. Öhberg, Europhys. Lett. 83, 54002 (2008).

[61] R. Gerritsma, G. Kirchmair, F. Zähringer, E. Solano, R. Blatt, and C. F. Roos, Nature (London) 463, 68 (2010).

[62] M. Aidelsburger, M. Atala, S. Nascimbene, S. Trotzky, Y.-A. Chen, and I. Bloch, Appl. Phys. B 113, 1 (2013).

[63] M. Gustavsson, E. Haller, M. J. Mark, J. G. Danzl, G. RojasKopeinig, and H.-C. Nägerl, Phys. Rev. Lett. 100, 080404 (2008).

[64] A. González-Tudela and J. I. Cirac, Phys. Rev. A 100, 053604 (2019). 